\title{
The In Vitro and In Vivo Inhibition of Protein Glycosylation and Diabetic Vascular Basement Membrane Thickening by Pyridoxal-5'-Phosphate
}

\author{
Michihiko HaYaKawA $^{1}$ and Masao ShiBATA ${ }^{2}$ \\ ${ }^{1}$ Nagoya Quarantine Station, Yokkaichi Branch Office, Ministry \\ of Health and Welfare, 5-1 Chitosecho, \\ Yokkaichi 510, Japan \\ ${ }^{2}$ The Department of Internal Medicine, Kakegawa City \\ General Hospital, Kakegawa, \\ Shizuoka, Japan
}

(Received September 27, 1990)

\begin{abstract}
Summary We studied the effect of pyridoxal-5'-phosphate (PAL-P) on protein glycosylation and diabetic nephropathy in NSY mice. In experiment 1 , an in vitro model of the browning phenomenon involving the incubation of lysine and glucose was inhibited by PAL-P. In experiment 2, administration of PAL-P to congenitally diabetic NSY mice markedly reduced the thickening of the glomerular basement membrane. These results suggest that PAL-P has the potential to be used for reducing the nephrotic complications of diabetes mellitus.

Key Words diabetic nephropathy, glycosylation, pyridoxal-5'-phosphate, NSY mouse, glomerular basement membrane, browning phenomenon, lysine, diabetic complications, Schiff's base, aldehyde
\end{abstract}

It has become clear that nonenzymatic glycosylation, which is the attachment of free sugars to certain amino acid residues of proteins, has a considerable degree of biomedical relevance, particularly in diabetes. It has been suggested that hyperglycemia, which promotes the increased nonenzymatic glycosylation of tissue proteins, contributes to the chronic complications of diabetes. Many of the proteins derived from specific tissues typically affected by diabetic complications, such as the glomerular basement membrane (1-6), have already been studied. If glycosylation is a causative factor in diabetic nephropathy, it has been suggested that pyridoxal5'-phosphate (PAL-P) might have a beneficial effect by virtue of its ability to compete with free glucose in the Schiff's reaction between the aldehyde and lysine residues of proteins. This study demonstrated the inhibitory effect of PAL-P on glycosylation in vitro, as well as the prevention of basement membrane thickening in the congenital diabetic mouse (NSY-mouse) $(7,8)$. 


\section{MATERIAL AND METHODS}

Experiment 1. The reaction mixture contained $50 \mathrm{~mm}$ lysine, $80 \mathrm{~mm}$ glucose, and $0.1 \mathrm{mM}$ sodium phosphate buffer ( $\mathrm{pH} 7.4$ ) with or without $0.1 \mathrm{mM}$ PAL-P in a final volume of $3 \mathrm{ml}$. It was incubated at $37^{\circ} \mathrm{C}$ for 7 days. All solutions were sterilized by passage through a millipore membrane filter. After incubation, the absorbance spectra was determined using a spectrophotometer (model UV160; Shimadzu, Kyoto).

Experiment 2. Congenitally diabetic NSY male mice $(n=5)$ were subcutaneously injected with PAL-P $(10 \mathrm{mg} / \mathrm{kg})$ every day for 8 weeks from 2 months of age. The same volume of physiological saline was administered to another group of NSY mice $(n=5)$. For the normal controls, the same amount of physiological saline was administered subcutaneously for the same period to $\mathrm{C} 57$ black mice from 2 months after birth $(n=5)$. All mice used in the experiment were precisely the same in age. Serum glucose, glycosylated hemoglobin $(\mathrm{GHb})$, serum fructosamine concentrations and the body weight were monitored. Blood was collected from the tail vein for determination of serum parameters. After 2 months of treatment, all mice were killed and the state of the glomerular basement membrane was investigated. Thickening of the basement membrane was determined as follows. By electron microscopy, 10 regions of the basement membrane were selected at random in a glomerulus, and the thickness of each region was measured. Measurement was performed for 10 glomeruli per mouse and all mice in each group were evaluated.

Statistics. Data are expressed as the mean \pm SD. Student's $t$-test was applied to determine the significance of differences between data for control and treated mice, with $p<0.05$ being taken as significant.

Chemicals. Lysine, glucose, and PAL-P were purchased from Sigma. The assay reagents for $\mathrm{GHb}$ and fructosamine were obtained from $\mathrm{GHb}$ Affin-system ISOLAB Inc. U.S.A. and Roche Corp. Switzerland, respectively.

\section{RESULTS}

Experiment 1. Inhibition of glycosylation by PAL-P in vitro (the browning phenomenon)

After incubation with lysine and glucose for 7 days, a unique pattern appeared in the absorption spectrum, consisting of a broad shoulder at about $330 \mathrm{~nm}$ which indicated the presence of the browning phenomenon (9).

The addition of PAL-P to the solution inhibited the occurrence of this phenomenon (Fig. 1). 


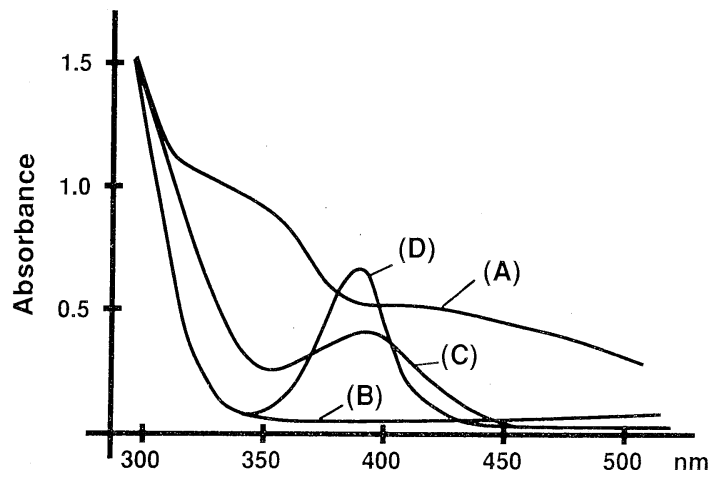

Fig. 1. Absorbance spectra of lysine and glucose incubated at $37^{\circ} \mathrm{C}$ for 7 days with or without PAL-P. (A) Post-incubation spectra of a mixture of $50 \mathrm{~mm}$ lysine and $80 \mathrm{~mm}$ glucose dissolved in $0.1 \mathrm{~mm}$ sodium phosphate buffer (pH 7.4). (B) Pre-incubation spectra of the same mixture as in (A). (C) Post-incubation spectra of a mixture of $50 \mathrm{~mm}$ lysine, $80 \mathrm{~mm}$ glucose, and $0.1 \mathrm{mM}$ PAL-P. (D) Pre-incubation spectra of the same mixture as in (C).

Experiment 2. Inhibition of protein glycosylation and basement membrane thickening by $P A L-P$ in vivo

1) Body weight. The body weight increased at the same rate in all groups, with no differences observed between the groups.

2) Serum glucose. The serum glucose concentrations of NSY mice administered PAL-P or physiological saline were much higher than the level in the normal control mice, but there was no significant difference between the two NSY groups (Fig. 2).

3) GHb. The GHb value was higher in both the PAL-P- and physiological

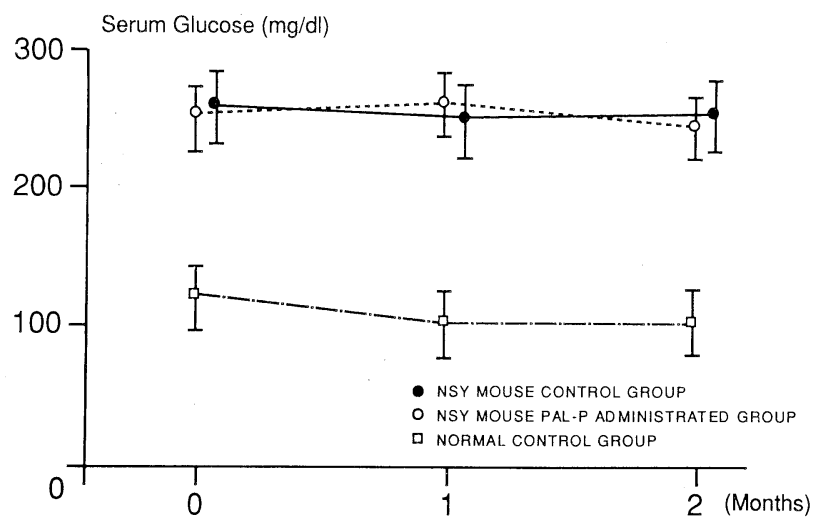

Fig. 2. Serum glucose concentrations. The serum glucose levels in the PAL-P- and physiological saline-treated groups were higher than those in the normal control group. However, no difference was observed between the groups of NSY mice. 
saline-treated groups of NSY mice than in the normal control mice. In the NSY mice receiving PAL-P, however, the GHb value decreased slightly after 8 weeks of administration $(p<0.1)$ (Fig. 3).

4) Fructosamine. The serum fructosamine level was much higher in the physiological saline-treated NSY mice than in the control mice, but in the PALP-treated NSY mice the level was similar to that in the normal control group. After 8 weeks of treatment, the difference between the PAL-P and saline groups of NSY mice was significant $(p<0.01)$ (Fig. 4).

5) Pathological features

Light microscopy: Periodic acid-Schiff staining showed diffuse glomerulo-

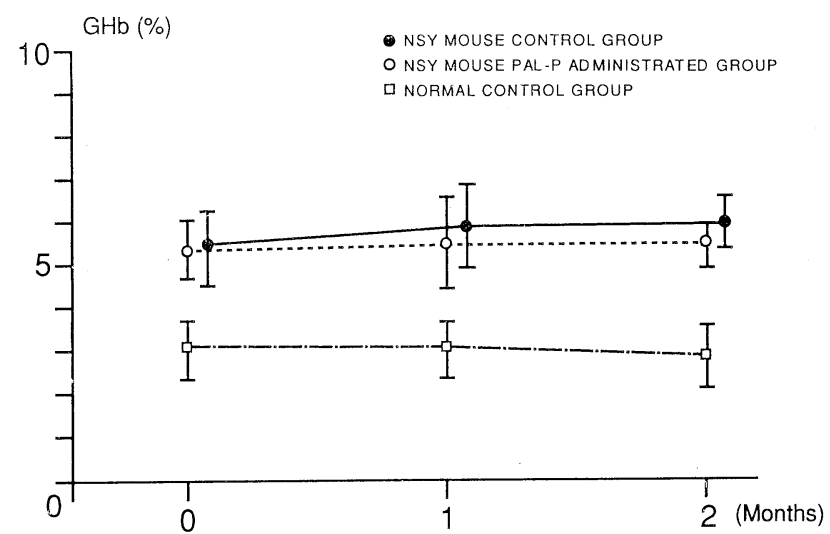

Fig. 3. Changes in GHb levels. The GHb levels in both NSY mouse groups were higher than in the normal control group, but in the PAL-P-treated group the mean value fell during the experimental period.

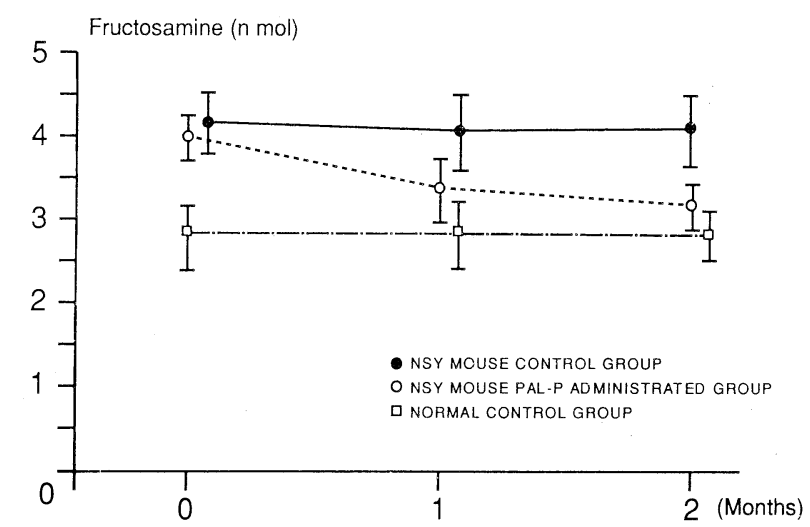

Fig. 4. Changes in serum fructosamine levels. The serum fructosamine was high in both NSY mouse groups, but in the PAL-P group, serum fructosamine was reduced and no significant difference was seen after 2 months administration. 
(A)

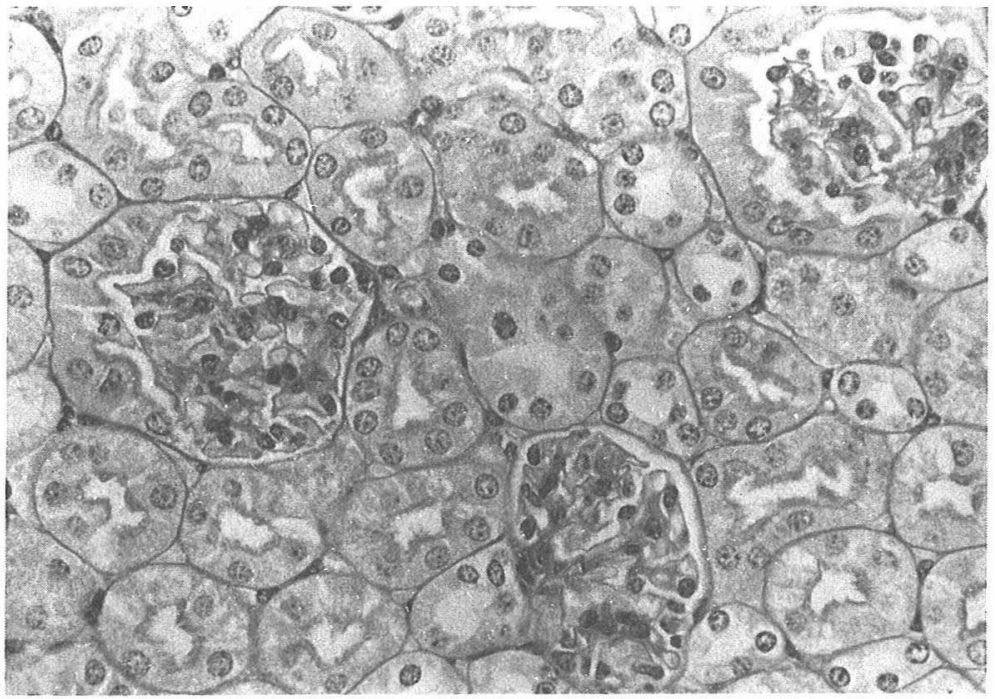

(B)

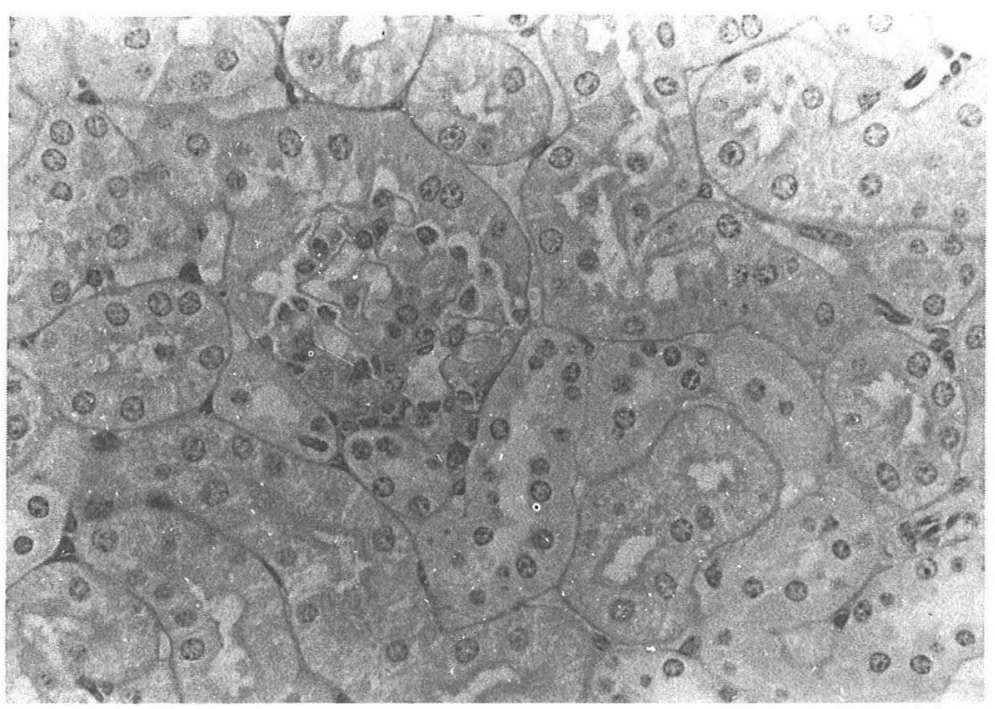

Fig. 5. (A) Light microscopic features of the renal glomeruli in a physiological saline-treated NSY mouse. Diffuse glomerulosclerosis and thickening of the basement membrane are seen (PAS stain $\times 400$ ). (B) Light microscopic features of the renal glomeruli in a PAL-P-treated NSY mouse. The extent of glomerulosclerosis and thickening of the basement membrane was reduced compared with the physiological saline-treated group (PAS stain $\times 400$ ). 
(A)

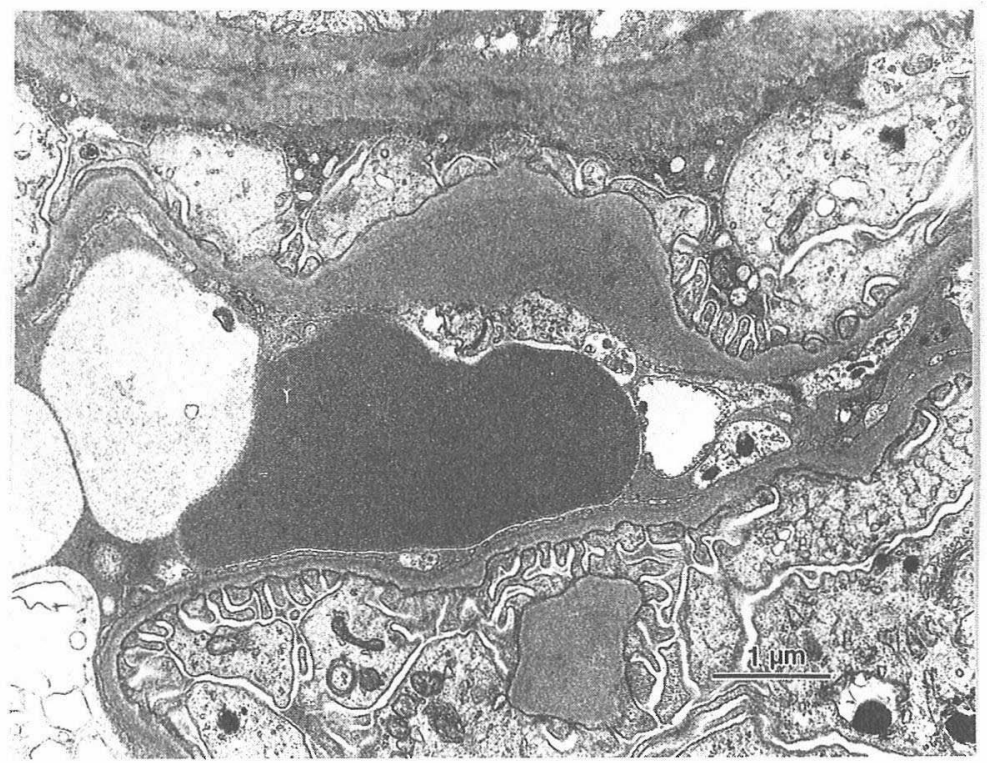

(B)

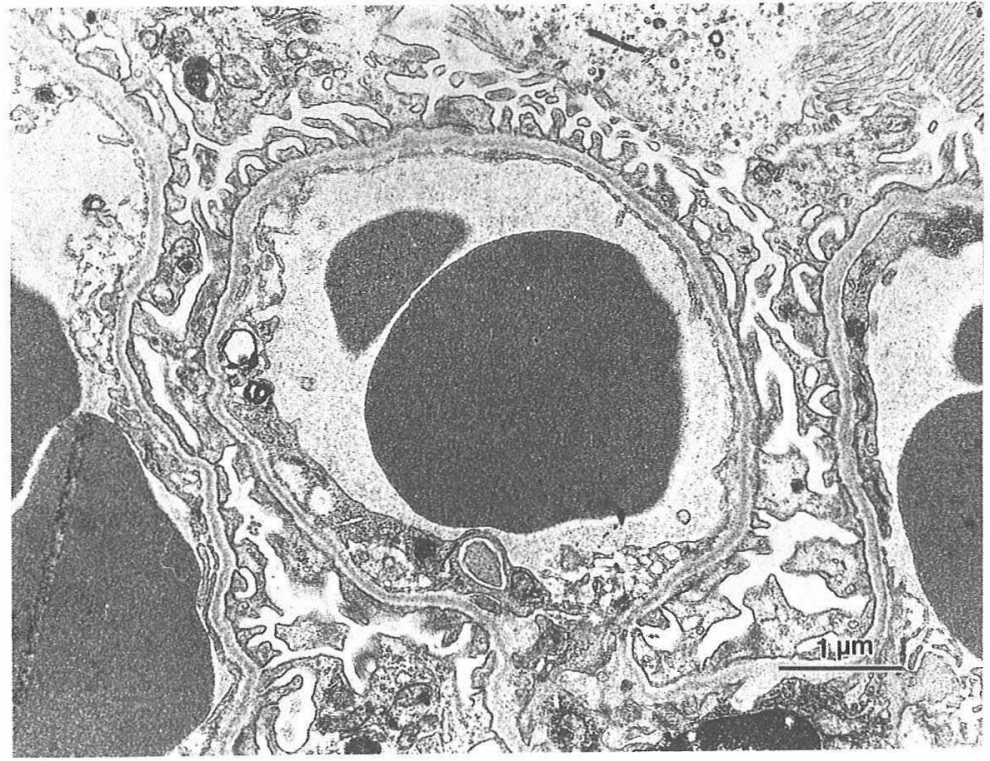

Fig. 6. (A) Electron microscopic features of the renal glomeruli in a physiological saline-treated NSY mouse. Increased mesangial matrix and irregular foot processes are seen. The glomerular basement membrane is segmentally thickened and homogeneous widening of the lamina densa is observed. (B) Electron microscopic features of the renal glomeruli in a PAL-P-treated NSY mouse. The features shown in (A) were markedly fewer in the PAL-P-treated NSY mice. No basement membrane thickening was observed. 


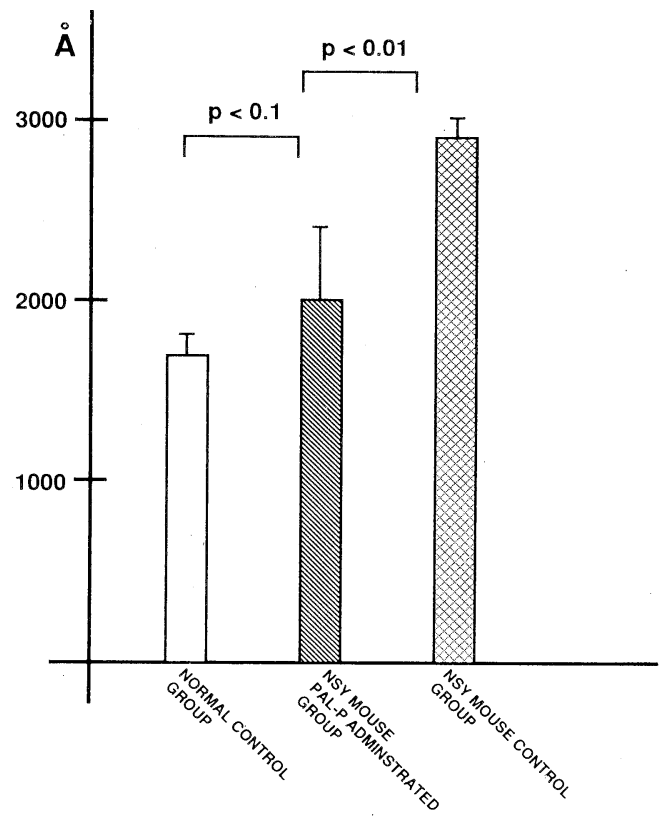

Fig. 7. Thickening of the glomerular basement membrane. Basement membrane thickening was reduced in the PAL-P treated NSY mice compared with the saline-treated NSY mice $(p<0.01)$.

sclerosis, an increase in the mesangial matrix, and thickening of the glomerular basement membrane in the physiological saline-treated group of NSY mice (Fig. 5(A)). The PAL-P-treated NSY mice, by contrast, had a similar histological appearance to the normal control mice (Fig. 5(B)).

Electron microscopy: In the physiological saline-treated NSY mice, an increase in the amount of mesangial matrix was noted by electron microscopy, and the matrix was seen to be aggregated into nodules. The glomerular basement membrane showed segmental thickening and its overall width was greater than normal. Homogeneous thickening of the lamina densa and the subendothelial accumulation of microfibrillar material were observed. Some podocytes had undergone degeneration and become detached from the underlying glomerular basement membrane (Fig. 6(A)). In the PAL-P-treated NSY mice, these features were extremely rare compared with the saline-treated mice (Fig. 6(B)). The thickness of the glomerular membrane was significantly greater in NSY mice compared to the control mice, but the PAL-P-treated NSY mice had significantly less thickening than saline-treated NSY mice $(p<0.01)$ (Fig. 7).

\section{DISCUSSION}

It has been reported that the injection of glycosylated plasma proteins pro- 
duces a pseudodiabetic thickening of the renal glomerular basement membrane in mice (10). The hypothesis that the non-enzymatic glycosylation of albumin might be a model reaction relevant to the pathogenesis of certain complications of chronic diabetes emerged with the demonstration that a variety of other proteins are also subject to glycosylation under physiologic conditions. This concept soon attracted the interest of investigators concerned with the biochemical basis of diabetic sequelae $(11,12)$. Many of the proteins from the tissues typically involved in diabetic complications, such as the glomerular basement membrane, have now been studied (1-6). Although non-enzymatic glycosylation also occurs under normal conditions, the chronic hyperglycemia characteristic of diabetes mellitus appears to be associated with an increase in the glycosylation process and thus with alteration in the function of circulating proteins, such as hemoglobin $(13,14)$, albumin $(15$, 16), and low-density lipoprotein (17-19). The kidney also appears to be affected by this process, since the increased non-enzymatic glycosylation of glomerular basement membrane collagen has been reported in both diabetic rats (20) and humans (21).

The pathological characteristics of the diabetic kidney are generally described as a thickened glomerular basement membrane and the presence of linear deposits of immunoglobulin, fibrin, complement factor C3 (22), and albumin (23) along the membrane. As the presence of immunoglobulins does not appear to be correlated with an active immunological process (24), the additional presence of nonimmunological proteins is suggestive of the secondary binding or trapping of protein by the glomerular basement membrane. Such a phenomenon could be due to an altered chemical composition or "reactivity" of the glomerular basement membrane and/or the proteins themselves. The non-enzymatic glycosylation of the glomerular basement membrane might offer an explanation for these changes occurring in diabetes. Various researchers have sought to find a means to inhibit, or at least reduce, this reaction. Ideally, this would be achieved by the optimization of glycemic control. Since this is still difficult to achieve, however, a second possible approach is pharmaceutical intervention. An important step in this direction has been the observation that aspirin can form an acetyl derivative by reacting with the same free amino acid group of the lysine residue that reacts with glucose (25). Aspirin can thus inhibit glycosylation by the rapid acetylation of this amino group (26), which prevents the initial formation of a Schiff's base. The use of aspirin as a glycosylation inhibitor, however, raises the problem of its side-effects, especially at the doses that would be required to obtain a significant level of inhibition. An alternative method to prevent the formation of damaging late glycosylation endproducts entails the use of aminoguanidine. This nucleophilic hydrazine compound has been shown to prevent the formation of late glycosylation endproducts and thus prevent the development of protein-protein cross links (27). Its inhibitory mechanism is different from that of aspirin and other acetylating compounds, since the latter inhibit the initial reaction between glucose and the protein amino groups, while aminoguanidine reacts irreversibly with the carbonyl group of the early 
glycosylation product. A third method for reducing the extent of non-enzymatic protein glycosylation is the use of aldehyde, which readily reacts with lysine in solution. We have shown previously that PAL-P could indeed reduce the in vitro non-enzymatic glycosylation of lysine and we also confirmed this finding in the case of the serum protein and hemoglobin of NSY mice in vitro. In this experiment the serum fructosamine level found to be decreased in the PAL-P-treated NSY mice indicated that the in vivo glycosylation of serum protein was also inhibited by PAL-P. When assessing fructosamine levels, numerous factors can interfere with the reaction of the reducing substances. To reduce inaccuracies, blood was collected just before injecting PAL-P into the mice. The half-life of PAL-P is only $1 \mathrm{~h}$ and it is readily excreted via the kidneys, so the PAL-P concentration would have been at its lowest level at that time. We also observed a reduction of glomerular basement membrane thickening in the PAL-P-treated NSY mice. High blood aldehyde levels may therefore have prevented a substantial amount of lysine residues from reacting with less accessible amino groups in the circulating and structural proteins. PAL-P is a water-soluble vitamin and excess intake is excreted via the kidneys, so its accumulation to toxic levels is rare.

In conclusion, optimization of glycemic control together with the early pharmacological inhibition of non-enzymatic glycosylation might reduce the incidence of diabetic nephropathy, but further studies of this subjects is needed.

\section{REFERENCES}

1) Lepape, A., Guitton, J. D., and Muh, J. P. (1981): Modification of glomerular basement membrane cross-links in experimental diabetic rats. Biochem. Biophys. Res. Commun., 100, 1214-1221.

2) Chang, A. Y., and Noble, R. E. (1980): 5-Hydroxymethylfurfural-forming proteins in the renal glomeruli of control and streptozotocin-diabetic rats. Life Sci., 26, 13291333.

3) Margo, P., Cohen, M. P., Urdanivia, E., Surma, M., and Wu, V. Y. (1980): Increased glycosylation of glomerular basement membrane collagen in diabetes. Biochem. Biophys. Res. Commun., 95, 765-769.

4) Cohen, M. P., and We, V.-Y. (1981): Identification of specific amino acid in diabetic glomerular basement membrane collagen subject to nonenzymatic glucosylation in vivo. Biochem. Biophys. Res. Commun., 100, 1549-1544.

5) Schliecher, E., and Wieland, O. H. (1984): Changes of human glomerular basement membrane in diabetes mellitus. J. Clin. Chem. Biochem., 22, 223-227.

6) Uitto, J., Perejda, A. J., Grant, G. A., Rowold, E. A., Kilo, C., and Williamson, J. R. (1982): Glucosylation of human glomerular basement membrane collagen: Increased content of hexose in ketoamine linkage and unaltered hydroxylysine- $O$-glycosides in patients with diabetes. Connect. Tissue Res., 10, 287-296.

7) Shibata, M. (1982): Microangiopathy in diabetic NSY mice, in Diabetic Microangiopathy; Proceeding of the International Symposium on Epidemiology of Diabetic Microangiopathy, ed. by Abe, H., and Hoshi, M., University of Tokyo Press., Tokyo, 
pp. $457-466$.

8) Shibata, M., and Yasuda, B. (1980): New experimental congenital diabetic mice (N.S.Y. mice). Tohoku J. Exp. Med., 130, 139-142.

9) Monnier, V. M., and Cerami, A. (1981): Nonenzymatic browning in vivo: Possible processes for aging of long-lived proteins. Science, 211, 491-493.

10) Mc. Verry, B. A., Hopp, A., Fisher, C., and Huehs, E. R. (1980): Production of pseudodiabetic renal glomerular changes in mice after repeated injection of glycosylated proteins. Lancet, 2, 738-740.

11) Koening, R. J., and Cerami, A. (1975): Synthesis of hemoglobin A1c in normal and diabetic mice: Potential model of basement membrane thickening. Proc. Natl. Acad. Sci. U.S.A., 72, 3687-3691.

12) Koening, R. J., and Cerami, A. (1980): Hemoglobin A1c and diabetes mellitus. Annu. Rev. Med., 31, 234-290.

13) Ditzel, J., Anderson, H., and Peters, N. D. (1975): Oxygen affinity of hemoglobin and red cell 2,3-diphosphoglycerate in childhood diabetes. Acta Pediatr. Scand., 64, 355361.

14) McDonald, M. J., Bleichman, M., and Bunn, H. F. (1979): Functional properties of the glycosylated minor components of human adult hemoglobin. J. Biol. Chem., 254, 702-707.

15) Williams, S. K., and Solenski, N. J. (1984): Enhanced vesicular ingestion of nonenzymatically glucosylated proteins by capillary endothelium. Microvasc. Res., 28, 311321.

16) Ghiggeri, G. M., Candiano, G., Delfino, G., Bianchini, F., and Queirolo, C. (1984): Glycosylated albumin and diabetic microalbuminuria: Demonstration of altered renal handling. Kidney Int., 25, 565-570.

17) Kim, H. J., and Kurup, I. V. (1982): Nonenzymatic glycosylation of human plasma low density lipoprotein: Evidence for in vitro and in vivo glycosylation. Metabolism, 31, 348-353.

18) Lorenzi, M., Cagliero, E., Markey, B., Henriksen, T., Witztum, J. L., and Sampietro, T. (1984): Interaction of human endothelial cells with elevated glucose concentrations and native and glycosylated low density lipoproteins. Diabetologia, 26, 218-222.

19) Kim, H. J., and Kurup, I. V. (1981): Decreased catabolism of glycosylated low density lipoprotein in diabetic rats. Diabetes, 30, 40A.

20) Cohen, M. P., Urdanivia, E., Surma, M., and Wu, V. Y. (1980): Increased glycosylation of glomerular basement collagen in diabetes. Biochem. Biophys. Res. Commun., 95, 765-769.

21) Uitto, J., Grant, C. A., Perejda, A. J., Rowald, E., and Williamson, J. R. (1980): Glycosylation of human glomerular basement membrane collagen (GBMC): Increased non-enzymatic glycosylation in diabetes. Fed. Proc., 39, 1972-1975.

22) Westberg, G. (1980): Diabetic nephropathy. Pathogenesis and prevention. Acta Endocrinol., 94 (Suppl 238), 85-101.

23) Michael, A. F., and Brown, D. M. (1981): Increased concentration of albumin in kidney basement membrane in diabetes mellitus. Diabetes, 30, 843-846.

24) Westberg, G., and Michael, A. F. (1972): Immunopathology of diabetic glomerular sclerosis. Diabetes, 21, 163-174.

25) Hawkins, D., Pinckard, R. N., Crawford, I. P., and Farr, R. S. (1969): Structural 
changes in human serum albumin induced by ingestion of acetylsalicylic acid. J. Clin. Invest., 48, 536-542.

26) Rendell, M., Nierenberg, J., Brannan, C., Velentine, J. L., Stephen, P. M., Dodds, S., Mercer, P., Smith, P. K., and Walder, J. (1986): Inhibition of glycation of albumin and hemoglobin by acetylation in vitro and in vivo. J. Lab. Clin. Med., 108, 286-293.

27) Brownlee, M., Vlassara, H., Kooney, A., Ulrich, P., and Cerami, A. (1986): Aminoguanidine prevents diabetes-induced arterial wall protein cross-linking. Science, 232, $1629-1632$. 